Canadian Science Publishing

Applied Physiology, Nutrition, and Metabolism Physiologie appliquée, nutrition et métabolisme

\title{
Effect of Exercise-Induced Dehydration on Circulatory Markers of Oxidative Damage and Antioxidant Capacity
}

\begin{tabular}{|c|c|}
\hline Journal: & Applied Physiology, Nutrition, and Metabolism \\
\hline Manuscript ID & apnm-2016-0701.R2 \\
\hline Manuscript Type: & Article \\
\hline Date Submitted by the Author: & 22-Jan-2017 \\
\hline Complete List of Authors: & $\begin{array}{l}\text { Georgescu, Vincent; Appalachian State University, Health and Exercise } \\
\text { Science } \\
\text { Souza-Junior, Tacito P.; Universidade Federal do Parana, Research Group } \\
\text { on Metabolism, Nutrition, and Strength Training } \\
\text { Behrens, Christian; University of Alabama at Birmingham } \\
\text { Barros, Marcelo; Universidade Cruzeiro do Sul, Institute of Physical Activity } \\
\text { and Sports Sciences (ICAFE) } \\
\text { Alves Bueno, Carlos; Universidade Federal do Parana, Research Group on } \\
\text { Metabolism, Nutrition, and Strength Training } \\
\text { Utter, Alan; Appalachian State University, } \\
\text { McAnulty, Lisa; Appalachian State University } \\
\text { McAnulty, Steven; Appalachian State University, }\end{array}$ \\
\hline Keyword: & Oxidative Stress, Dehydration, Antioxidants, exercise, Humans \\
\hline
\end{tabular}

SCHOLARONE $^{\text {M }}$

Manuscripts 
Title: Effect of Exercise-Induced Dehydration on Circulatory Markers of Oxidative Damage and Antioxidant Capacity

Running Title: Dehydration, Plasma Oxidative Stress, and Antioxidant Capacity

Authors: Vincent P. Georgescu ${ }^{1}$, Tacito P. de Souza Junior ${ }^{1,3}$, Christian Behrens ${ }^{2}$, Marcelo P. Barros $^{4}$, Carlos Alves Bueno ${ }^{3}$, Alan C. Utter ${ }^{1}$ (FACSM), Lisa S. McAnulty ${ }^{2}$, and Steven R. McAnulty ${ }^{1}$.

Institutions: ${ }^{1}$ Department of Health and Exercise Science and ${ }^{2}$ Department of Nutrition and Health Care Management, Appalachian State University, Boone, NC USA. ${ }^{3}$ Universidade Federal do Parana, Research Group on Metabolism, Nutrition, and Strength Training, Curitiba, Brazil. ${ }^{4}$ Postgraduate program in Human Movement Sciences, Institute of Physical Activity and Sports Sciences (ICAFE), Cruzeiro do Sul University, Sao Paulo, Brazil.

\section{Communication:}

Steven R. McAnulty, Ph.D.

Dept HES, PO Box 32071, Appalachian State University, Boone,

NC 28608; 828-262-7151 (phone); 828-262-3138 (fax);

mcanltysr@appstate.edu

Journal: Applied Physiology Nutrition and Metabolism 


\begin{abstract}
Dehydration is a common event associated with exercise. However, few studies have examined the effects of dehydration on plasma redox status in humans. Eighty-two athletes were recruited and had Baseline anthropometrics and blood samples obtained. Athletes then engaged in a dehydration protocol, training until $3 \%$ of pre-weight body mass was lost. Athletes returned to the lab and had Post-dehydration blood collected. Athletes then consumed an isotonic drink until pre-exercise body weight was reestablished. Blood was then recollected (1-h post-full rehydration (PFR)). Samples were centrifuged and the plasma snap frozen in liquid nitrogen and stored at $-80^{\circ} \mathrm{C}$. Lipid and protein oxidative stress was determined by measuring $\mathrm{F}_{2}-$ isoprostanes and protein carbonyls (PC), respectively. Antioxidant capacity was determined by the ferric reducing ability of plasma (FRAP) and trolox equivalent antioxidant capacity (TEAC) assays. Plasma osmolality was determined using an osmometer. Statistical analysis utilized a 1way ANOVA with post-hoc testing. Values are reported as mean $\pm \mathrm{SD}$. Plasma osmolality was significantly elevated immediately post-dehydration $(\mathrm{p} \leq 0.001)$ but decreased to Baseline at PFR. Plasma TEAC increased immediately post-dehydration and at PFR $(\mathrm{p} \leq 0.001)$. FRAP increased immediately post-dehydration $(\mathrm{p} \leq 0.001)$ and decreased to below Baseline at PFR $(\mathrm{p} \leq 0.05)$. Conversely, $\mathrm{F}_{2}$-isoprostanes declined significantly from Baseline to immediately postdehydration and then significantly rose at PFR $(\mathrm{p} \leq 0.001)$, while PC declined at PFR $(\mathrm{p} \leq 0.01)$. This study indicates that dehydration and exercise cause a significant increase in plasma osmolality and antioxidant potential immediately post-exercise. We propose dehydration significantly elevates antioxidant concentration which suppresses $\mathrm{F}_{2}$-isoprostanes and $\mathrm{PC}$. Key Words: Oxidative Stress, Dehydration, Antioxidants, Humans, Exercise
\end{abstract}




\section{Introduction}

Oxidative stress during exercise is known to be involved in many adverse outcomes which can often result in subsequent damage to cellular proteins, lipids, and nucleic acids (Powers et al. 2016; Sallam and Laher 2016). Additionally, exercise is often associated with dehydration and investigations in vitro have revealed that dehydration and hyperthermia elicit increased cellular and oxidative stress. Situations involving heat exposure and dehydration are associated with ischemia/reperfusion events that are highly damaging to most mammals (King et al. 2016). However, research in vivo utilizing specific and sensitive biomarkers of oxidative stress related to different physiological components such as lipids and proteins is more limited (Penkman et al. 2008; Paik et al. 2009; Hillman et al. 2011). While oxidative stress is typically linked to increases in oxygen consumption which result in formation of reactive species (Powers et al. 1999), the specific impact of dehydration on oxidative stress in exercising humans is less known. Animals subjected to environmental and metabolic stressors elicit certain response patterns. The most commonly observed response is an eventual enhancement of antioxidant defenses (Hermes-Lima and Zenteno-Savin 2002).

Alterations in cellular hydration not only contribute to changes in metabolic pathways, but also determine the cellular response to various forms of stress. Whereas cellular swelling triggers anabolic pathways and protects cells from oxidative damage, cellular dehydration contributes to catabolism and increases the cellular susceptibility to damage. Adaptations via accumulation of osmolytes and the expression of heat shock proteins provide cellular tolerance to hyper-osmolality under dehydrating conditions. Therefore, there appears to be a close relationship between osmotic and oxidative stresses (Schliess and Haussinger 2002). Beyond the theoretical, McAnulty et al. (McAnulty et al. 2005a) examined the effect of exercising at a fixed 
intensity $\left(50 \%\right.$ of $\left.\mathrm{VO}_{2 \max }\right)$ in a hot environment $\left(35^{\circ} \mathrm{C}, 70 \%\right.$ relative humidity $\left.(\mathrm{RH})\right)$ versus exercising in a cool environment $\left(25^{\circ} \mathrm{C}, 40 \% \mathrm{RH}\right)$ on markers of oxidative stress. The heat stress trial produced a core temperature of $39.5^{\circ} \mathrm{C} . \mathrm{F}_{2}$-isoprostanes increased in both groups following exercise but were significantly higher in the hot environment. Dehydration was also greater in the hot trial $(2.8 \%$ of body weight $)$ compared to the neutral trial $(0.6 \%$ of body weight). However, this study did not examine the effects of antioxidant status or the effect of rehydration on $\mathrm{F}_{2}$-isoprostanes. Interestingly, Laitano et al. (Laitano et al. 2010) found that in euhydrated humans heat stress or exercise alone did not alter GSH, SOD activity, or increase plasma isoprostanes. However, combined heat stress and exercise increased both GSH and GSSG, decreased SOD activity, but did not alter isoprostanes. This suggests that heat stress, independently of dehydration, increases antioxidant defense which may compensate for the effects of heat stress-induced oxidative stress. (Laitano et al. 2010) .

Therefore, given the lack of an existing comprehensive examination of the effects of precisely monitored dehydration on specific indices of oxidative stress and antioxidant responses, the objective of this study was to investigate the effects of dehydration and rehydration by using sensitive indirect measures of oxidative stress and the antioxidant capacity response. We hypothesized that dehydration would increase oxidative stress and antioxidant capacity.

\section{METHODS}

\section{Subjects}

Fifty-Six male collegiate wrestlers and 26 female $(\mathrm{N}=82)$ collegiate soccer players were recruited to undergo an acute dehydration (3\% body weight for males and females) and rehydration protocol. Due to other study obligations and dropouts, the subject numbers for 
plasma osmolality and oxidant and antioxidant assays are different. While plasma osmolality was obtained for 80 subjects, plasma availability resulted in different numbers for the other assays as listed in the Figure Legends. Written informed consent was obtained from each subject, and the experimental procedures were approved by the institutional review board of Appalachian State University.

\section{Research Design}

The experiment followed a repeated measures design with all subjects serving as their controls. Subjects were instructed to report to the Appalachian State University (ASU) Human Performance Laboratory (HPL) in a hydrated state at 8:00 a.m. Subjects proceeded to follow a series of stations in the following order: urine sample, body mass, blood samples. This order was followed at baseline, immediately post-dehydration, and one-hour post-full rehydration (PFR) time points.

After completing baseline measurements, the athletes were assigned a mass loss goal of $3 \%$ body mass $(\mathrm{BM})$ for males and females. The participants then underwent a supervised exercise regimen of two hours to induce the prescribed mass loss. A Tanita TBF 300 digital scale (Tanita Arlington Heights, IL 60005) sensitive to 0.1 percent and $0.1 \mathrm{~kg}$ was used to determine percent body fat and body mass, respectively. The male wrestling regimen incorporated an initial warmup of jogging and gymnastic maneuvers such as cartwheels and round offs, followed by several stretching exercises. The remainder of the dehydration protocol involved drilling. Drills included from the feet (stance, defense, changing levels) and from the mat (standups, short sit, Granby shrug). This training occurred indoors with a temperature of $22.7^{\circ} \mathrm{C}$ and $53 \%$ relative humidity. The soccer training involved attacking, crossing and finishing, overlapping runs, and defending. This training occurred outside with a temperature of 
$13.3^{\circ} \mathrm{C}$ and $78 \%$ relative humidity. Scales were available at all times for the subjects to check if they had attained their mass loss goal.

Specific dehydration data have been previously published (Sommerfield et al. 2015). Upon exercise completion, subjects returned to the HPL where they followed the same measurement order as baseline. Once post-exercise measures were completed, subjects were given an electrolyte-carbohydrate beverage $\left(6 \%\right.$ or $60 \mathrm{~g} \cdot \mathrm{L}^{-1}$; Gatorade ${ }^{\circledR}$, Barrington, IL, USA). The electrolyte-carbohydrate beverage contained $20 \mathrm{mmol} \cdot \mathrm{L}^{-1}$ sodium and $3.2 \mathrm{mmol} \cdot \mathrm{L}^{-1}$ of potassium among other minerals. The amount of beverage assigned was equal to the body mass lost by each subject during exercise. All fluids were precisely measured and provided to subjects by research assistants. Each subject was required to consume fluids equivalent to half of the BM lost during the dehydration protocol within the first $20 \mathrm{~min}$ of the rehydration protocol. The second half of the lost BM was consumed from minutes 21 to 40 of the rehydration protocol. Once pre-exercise BM was attained, the final blood samples were collected 1-hour PFR, corresponding to two hours post-commencement of fluid consumption.

\section{Blood Sampling Conditions and Disposition}

With subjects in the seated position, blood samples were drawn from an antecubital vein into sodium heparin tubes and centrifuged at 3,500 RPM, $4^{\circ} \mathrm{C}$ x $10 \mathrm{~min}$. Plasma was aliquoted, snap frozen in liquid $\mathrm{N}_{2}$, and stored at $-80^{\circ} \mathrm{C}$ until analysis for plasma osmolality, $\mathrm{F}_{2}$ isoprostanes, protein carbonyls, and the following plasma antioxidant parameters: Ferric Reducing Ability of Plasma (FRAP) and Trolox Equivalent Antioxidant Capacity (TEAC). $F_{2}$-isoprostanes

$\mathrm{F}_{2}$-isoprostanes were measured using the 8-Isoprostane Express ELISA Kit (Item № 516360) from Cayman Chemical (Ann Arbor, MI USA). It is not uncommon for ELISA 
techniques to measure higher concentrations, compared to LC and GC-MS technologies, due to cross reactivity with similarly structured molecules versus the parent molecule. We performed a de-esterification protocol to measure total $F_{2}$-isoprostanes versus free $F_{2}$-isoprostanes by adding $15 \%$ potassium hydroxide to all samples and incubating at $40^{\circ} \mathrm{C}$ for $60 \mathrm{~min}$. Samples were then neutralized by addition of $1 \mathrm{M}$ potassium phosphate, $\mathrm{pH} 7.0-7.4$, then purified by solid phase extraction on C18 cartridges (Sep-Pak Plus silica cartridges, Waters) and ultimately reacted with antibodies provided in the kit to determine total $\mathrm{F}_{2}$-isoprostanes. A 4-parameter logistic fit curve was used to determine final concentrations. Intra-assay and inter-assay coefficients of variation were less than $6 \%$ and $4 \%$, respectively.

\section{Protein Carbonyls}

Protein Carbonyls were measured using the Protein Carbonyl Colorimetric Assay Kit by Cayman Chemical (Item № 10005020). The level of plasma protein oxidative damage, indicated by the protein carbonyl content (Cadenas et al. 2016), was measured according to the method of Levine et al. (McDougall et al. 2016), based on derivatization of carbonyls with DNPH as described in detail by Yan and Sohal (Mah et al. 2015). The isolated protein was counted in a Beckman scintillation counter and calculated using a standard curve. Intra-assay and inter-assay coefficients of variation were less than $8 \%$ and $9 \%$, respectively.

\section{Ferric Reducing Antioxidant Potential (FRAP)}

Total plasma antioxidant potential was determined by the ferric reducing ability of plasma (FRAP) assay according to the methodology of Benzie et al. (Benzie and Strain 1996). The basis of this assay is that water soluble reducing agents (antioxidants) in the plasma will reduce ferric ions to ferrous ions which then react with an added chromogen. Samples and standards were analyzed in duplicate and expressed as ascorbate equivalents based on an 
ascorbate standard curve $(0-1000 \mu \mathrm{mol})$. Intra-assay and inter-assay coefficients of variation were less than 5\% and 7\%, respectively.

\section{Trolox Equivalent Antioxidant Capacity (TEAC)}

Antioxidant activity was further determined in EDTA treated plasma samples using the TEAC method as described by Villano et al. (Villano et al. 2004). Briefly, a free radical producing enzymatic system was created using the horseradish peroxidase enzyme and $2,2^{\prime}$ azinobis (3-ethylbenzothiazoline-6-sulfonic acid) (ABTS). This radical is produced by a reaction with $1.5 \mathrm{mM}$ ABTS, $15 \mu \mathrm{M}$ hydrogen peroxide and $0.25 \mu \mathrm{M}$ peroxidase in $50 \mathrm{mM}$ glycine-HCL buffer ( $\mathrm{pH} 4.5)$. One hundred $\mu \mathrm{L}$ of sample were placed in individual cuvettes, and two $\mathrm{mL}$ of ABTS were then added. After two minutes of incubation, the wavelength of the solution was measured at $414 \mathrm{~nm}$. All samples were tested in duplicate. The decrease in absorbance was quantified using a linear regression model of an eight-point standard curve $(0-200 \mu \mathrm{M})$ and expressed as Trolox equivalents. Intra-assay and inter-assay coefficients of variation were less than $5 \%$ and $3 \%$, respectively.

\section{Plasma Osmolality}

Blood samples were obtained to test for plasma osmolality (mOsm) using a freezing point depression osmometer (Model 3250, Advanced Instruments Inc., Norwood, MA, USA).

\section{Statistical Analysis}

Data were analyzed using a 1-way ANOVA with post hoc testing using Instat statistical software (GraphPad StatMate version 2.00 for Windows, GraphPad Software, San Diego CA, USA). Data are expressed as mean \pm SD. All data were screened for outliers with outliers designated as being outside of the mean $\pm 2.5 \mathrm{SD}$.

\section{Results}


Subject baseline characteristics are summarized in Table 1. Outcome data for male and female athletes are presented together for each variable. Males were taller and heavier and had less body fat than females. Females had more years of experience than their male counterparts. Plasma osmolality (mOsm, $\mathrm{n}=80$ ) was significantly elevated immediately post-exercise (pre: $279.0 \pm 14.3$, post: $286.2 \pm 15.9, \mathrm{p} \leq 0.01)$ but returned to near normal values $(279.42 \pm 15.4)$ post-full rehydration (PFR) (Figure 1). Plasma osmolality data were used to indicate dehydration state and have been previously published by Sommerfield et al. 2015 (Sommerfield et al. 2015). Plasma TEAC ( $\mu \mathrm{mol} / \mathrm{L}$ trolox equivalents, $n=33)$ exhibited the same pattern of rise from baseline to immediately post-dehydration followed by a fall PFR (pre: $498.06 \pm 6.02$, post: $552.2 \pm 12.11$, PFR: $513.5 \pm 1.85)$ and displayed statistical significance pre to immediate post $(\mathrm{p} \leq 0.001)$ and pre to PFR ( $\mathrm{p} \leq 0.001)$ (Figure 2). Plasma FRAP ( $\mu \mathrm{mol} / \mathrm{L}$ ascorbate equivalents, $\mathrm{n}=76)$ values also increased immediately post-dehydration (pre: $100.85 \pm 8.65$, post: $140.31 \pm 17.2, p \leq 0.001$ ) and decreased to below baseline levels PFR $(95.3 \pm 9.6, \mathrm{p} \leq 0.05)$ (Figure 3). Protein carbonyls $(\mathrm{ng} / \mathrm{mL} \mathrm{n}=24)$ declined immediately post-dehydration (pre: $32.3+4.9$, post: $31.3 \pm 5.7)$ but did not rebound at PFR $(\mathrm{p} \leq 0.01)$ (Figure 4). Mean concentrations of $\mathrm{F}_{2}$-isoprostanes $(\mathrm{pg} / \mathrm{mL}, \mathrm{n}=24)$ declined from baseline to immediately post-dehydration (pre: $437.6 \pm 125.5$, post: $77.5 \pm 49.6$, $\mathrm{p} \leq 0.001)$ and then rose to $(699.7 \pm 154.2)$ PFR $(\mathrm{p} \leq 0.001)$ (Figure 5). Significant negative correlations existed between $\mathrm{F}_{2}$-isoprostanes and FRAP at PFR $(\mathrm{p} \leq 0.005$, correlation $=-0.587)$. Weak correlations were also displayed between plasma osmolality and TEAC immediately postdehydration $(\mathrm{p} \leq 0.02$, correlation $=-0.404)$ and PFR $(\mathrm{p} \leq 0.037$, correlation $=-0.376)$ (Table 2$)$.

\section{Discussion}

This study indicates that dehydration, coupled with exercise, increases plasma osmolality and antioxidant potential immediately post-dehydration which results in a decrease in oxidative 
damage. Interestingly, our study also found that $3 \%$ dehydration results in deviating antioxidant and oxidative stress responses post-rehydration. Furthermore, there is also a divergence between lipid peroxidation and protein oxidation once rehydration is re-established. A possible limitation of our study is that we did not have a control group that only performed exercise without dehydration or dehydration with no exercise. However, in support of our choice to not utilize these control groups, Hillman et al. (Hillman et al. 2011) found the presence of dehydration alone to be a consistent factor with disruption of the redox state. Hillman et al. (Hillman et al. 2011) examined the effects of exercise-induced dehydration with and without hyperthermia on oxidative stress. Seven healthy male, trained cyclists completed 90 minutes of cycling exercise at $95 \%$ of lactate threshold followed by a $5-\mathrm{km}$ time trial in 4 trials: (1) euhydration in a warm environment), (2) dehydration in a warm environment (3) euhydration in a thermoneutral environment, and (4) dehydration in a thermoneutral environment. Oxidized glutathione (GSSG) increased significantly post-exercise in dehydration trials only, suggesting that dehydration can solely disrupt antioxidant defenses. Further, maintenance of euhydration attenuated these increases regardless of environmental condition.

In the current investigation, we found a suppression of $\mathrm{F}_{2}$-isoprostanes immediately after dehydration, followed by an elevation post-full rehydration in contrast to exercise studies that did not incorporate dehydration (McAnulty et al. 2005b; McAnulty et al. 2007a; McAnulty et al. 2007b). Quantification of $\mathrm{F}_{2}$-isoprostanes provides an accurate assessment of oxidative damage both in vitro and in vivo. A National Institutes of Health-sponsored independent study found $\mathrm{F}_{2}$ isoprostanes to be the most reliable index of in vivo oxidant damage, when compared against other well-known biomarkers (Milne et al. 2007). It has been suggested that oxidant and antioxidant changes observed in the blood during exercise are dependent on temperature and 
fluid balance. Possible mechanisms linking exercise with these components included shear stress, hemo-concentration, and signaling pathways involving muscle osmoregulation. These authors concluded that multiple mechanisms may be responsible for the changes in the oxidant/antioxidant balance in the blood during exercise, reflecting requirements of adapting to osmotic and hyperthermia challenges (King et al. 2016). We observed that modest dehydration also results in changes in these factors. Nonetheless, the long-term and detailed effects of training on blood rheology have not been fully determined (El-Sayed et al. 2005).

Hillman et al. (Hillman et al. 2013) examined glycerol hyper-hydration on levels of preand post-exercise oxidative stress. In all trials, total glutathione increased post-exercise while oxidized glutathione and protein carbonyl concentrations increased post-exercise for the control trial only. The conclusion was that fluid intake attenuated oxidative damage but did not enhance thermoregulation or performance. Furthermore, Paik et al. (Paik et al. 2009) examined the effects of hydration status on oxidative DNA damage and exercise performance and determined euhydration was beneficial at preventing oxidative damage. Dehydration significantly reduced time to exhaustion and increased plasma MDA levels compared to Control. Additionally, dehydration significantly increased oxidative DNA damage during exercise, but fluid replacement with water or sports drink nullified the effect equally.

We found that protein carbonyls decreased similarly to $\mathrm{F}_{2}$-isoprostanes immediately postdehydration but not to the same extent. A relationship may exist between lipid modification and modification of proteins in conditions of oxidative stress. Oxidized polyunsaturated fatty acids (PUFAs) readily bind to protein nucleophiles and can be derivatized by 2, 4dinitrophenylhydrazine (DNPH) and constitute significant DNPH-detectable protein-bound carbonyl activity that serves as a key indicator of oxidative stress in tissues (Sayre et al. 2006). 
Plasma protein carbonyls are abundant and easily detectable markers of oxidative stress that are widely used for the interpretation of exercise-induced changes in redox balance (Wadley et al. 2016). Despite many studies reporting acute increases in protein carbonyl concentration in response to exercise, some studies have shown decreases similar to our observations in the current investigation. Intensity, duration, and novelty of the exercise bout may all influence the protein carbonyl response to exercise (Wadley et al. 2016).

We also observed increases in both FRAP and TEAC responses immediately postdehydration with values returning to baseline by 1-hour PFR. We further propose that the elevation in post-exercise FRAP and TEAC antioxidant values immediately post-dehydration were sufficient to reduce the formation of $\mathrm{F}_{2}$-isoprostanes and protein carbonyls. What is unexplained is the divergent response we observed immediately post-rehydration between $\mathrm{F}_{2}$ isoprostanes and protein carbonyls. $\mathrm{F}_{2}$-isoprostanes increased while protein carbonyls returned to normal. This suggests differences in formation mechanisms between the two.

In support of our hypothesis that increased antioxidant responses diminished oxidative damage post-dehydration, Knez et al. (Knez and Periard 2014) examined the impact of changes in oxidative stress and antioxidant status in response to playing tennis in HOT and COOL conditions. Core body temperature, body mass, indirect markers of oxidative stress, and antioxidant status were assessed. Oxidative stress markers remained consistent through play and into recovery. Tennis in the COOL had no impact on antioxidant status. However, antioxidant status increased significantly in the HOT compared with the COOL environment. Body mass losses were similar between conditions, but rectal temperature increases were greater in the HOT versus COOL environment. The authors concluded that tennis in the heat did not exacerbate the development of oxidative damage but significantly increased antioxidant status. In support of 
our study conclusions, it was suggested that the heat stress observed in the HOT environment provided an up-regulation of antioxidant defense which dampened cellular damage. Also, Laitano et al. (Laitano et al. 2012) found that during exercise, active skeletal muscles release GSH into the circulation under moderate dehydration and subsequent rehydration, possibly to enhance the antioxidant defense. An increase in the immune response may also be a counter to increased oxidative stress in conditions of hyperthermia and dehydration (Mestre-Alfaro et al. 2012; Sureda et al. 2015).

In conclusion, our study indicates that dehydration, coupled with exercise, causes increases in plasma osmolality and antioxidant potential which initially suppress lipid and protein oxidative damage markers followed by an increase after rehydration in $\mathrm{F}_{2}$-isoprostanes but not protein carbonyls. We believe this is the first study to report a divergent response to lipid and protein oxidation after dehydration followed by rehydration. Our findings further suggest that dehydration results in a cellular stress that is not completely resolved after rehydration. The specific mechanisms concerning the divergence between protein and lipid oxidation following rehydration should be further examined in future studies.

\section{Conflict of Interest Disclaimer}

The authors report no conflicts of interest with this manuscript.

Acknowledgements : Supported by a grant from Artann Laboratories, Inc. Grant No. 2R44

AG042990 (CFDA 93.866). Dr. Tácito P. Souza-Junior, is supported by a CNPq fellowship \#216382/2014-9 


\section{References}

Benzie, I.F. and Strain, J.J. 1996. The ferric reducing ability of plasma (FRAP) as a measure of "antioxidant power": the FRAP assay. Anal. Biochem. 239: 70-610.1006/abio.1996.0292.

Cadenas, E., Packer, L., and Traber, M.G. 2016. Antioxidants, oxidants, and redox impacts on cell function - A tribute to Helmut Sies. Arch. Biochem. Biophys. 595: 94-

910.1016/j.abb.2015.11.012.

El-Sayed, M.S., Ali, N., and El-Sayed Ali, Z. 2005. Haemorheology in exercise and training. Sports Med. 35: 649-70

Hermes-Lima, M. and Zenteno-Savin, T. 2002. Animal response to drastic changes in oxygen availability and physiological oxidative stress. Comp. Biochem. Physiol. C. Toxicol.

Pharmacol.133: 537-56

Hillman, A.R., Turner, M.C., Peart, D.J., Bray, J.W., Taylor, L., McNaughton, L.R., et al. 2013. A comparison of hyperhydration versus ad libitum fluid intake strategies on measures of oxidative stress, thermoregulation, and performance. Res. Sports Med. 21: 305$1710.1080 / 15438627.2013 .825796$.

Hillman, A.R., Vince, R.V., Taylor, L., McNaughton, L., Mitchell, N., and Siegler, J. 2011. Exercise-induced dehydration with and without environmental heat stress results in increased oxidative stress. Appl. Physiol. Nutr. Metab. 36: 698-70610.1139/h11-080.

King, M.A., Clanton, T.L., and Laitano, O. 2016. Hyperthermia, dehydration, and osmotic stress: unconventional sources of exercise-induced reactive oxygen species. Am. J. Physiol. Regul. Integr. Comp. Physiol. 310: R105-1410.1152/ajpregu.00395.2015. 
Knez, W.L. and Periard, J.D. 2014. The impact of match-play tennis in a hot environment on indirect markers of oxidative stress and antioxidant status. Br. J. Sports Med. 48 Suppl 1: i596310.1136/bjsports-2013-093248.

Laitano, O., Kalsi, K.K., Pearson, J., Lotlikar, M., Reischak-Oliveira, A., and Gonzalez-Alonso, J. 2012. Effects of graded exercise-induced dehydration and rehydration on circulatory markers of oxidative stress across the resting and exercising human leg. Eur. J. Appl. Physiol. 112: 1937-4410.1007/s00421-011-2170-2.

Laitano, O., Kalsi, K.K., Pook, M., Oliveira, A.R., and Gonzalez-Alonso, J. 2010. Separate and combined effects of heat stress and exercise on circulatory markers of oxidative stress in euhydrated humans. Eur. J. Appl. Physiol. 110: 953-6010.1007/s00421-010-1577-5.

Mah, E., Sapper, T.N., Chitchumroonchokchai, C., Failla, M.L., Schill, K.E., Clinton, S.K., et al. 2015. alpha-Tocopherol bioavailability is lower in adults with metabolic syndrome regardless of dairy fat co-ingestion: a randomized, double-blind, crossover trial. Am. J. Clin. Nutr. 102: 1070-8010.3945/ajen.115.118570.

McAnulty, S.R., Hosick, P.A., McAnulty, L.S., Quindry, J.C., Still, L., Hudson, M.B., et al. 2007a. Effect of pharmacological lowering of plasma urate on exercise-induced oxidative stress. Appl. Physiol. Nutr. Metab. 32: 1148-5510.1139/H07-131.

McAnulty, S.R., McAnulty, L., Pascoe, D.D., Gropper, S.S., Keith, R.E., Morrow, J.D., et al. 2005a. Hyperthermia increases exercise-induced oxidative stress. Int. J. Sports Med. 26: 188$9210.1055 / \mathrm{s}-2004-820990$.

McAnulty, S.R., McAnulty, L.S., Nieman, D.C., Morrow, J.D., Shooter, L.A., Holmes, S., et al. 2005b. Effect of alpha-tocopherol supplementation on plasma homocysteine and oxidative stress 
in highly trained athletes before and after exhaustive exercise. J. Nutr. Biochem. 16: 530710.1016/j.jnutbio.2005.02.001.

McAnulty, S.R., Owens, J.T., McAnulty, L.S., Nieman, D.C., Morrow, J.D., Dumke, C.L., et al. 2007b. Ibuprofen use during extreme exercise: effects on oxidative stress and PGE2. Med. Sci. Sports Exerc. 39: 1075-910.1249/mss.0b13e31804a8611.

McDougall, M.Q., Choi, J., Stevens, J.F., Truong, L., Tanguay, R.L., and Traber, M.G. 2016. Lipidomics and $\mathrm{H} 2(18) \mathrm{O}$ labeling techniques reveal increased remodeling of DHA-containing membrane phospholipids associated with abnormal locomotor responses in alpha-tocopherol deficient zebrafish (danio rerio) embryos. Redox Biol. 8: 165-7410.1016/j.redox.2016.01.004. Mestre-Alfaro, A., Ferrer, M.D., Banquells, M., Riera, J., Drobnic, F., Sureda, A., et al. 2012. Body temperature modulates the antioxidant and acute immune responses to exercise. Free Radic. Res. 46: 799-80810.3109/10715762.2012.680193.

Milne, G.L., Yin, H., Brooks, J.D., Sanchez, S., Jackson Roberts, L., 2nd, and Morrow, J.D. 2007. Quantification of F2-isoprostanes in biological fluids and tissues as a measure of oxidant stress. Methods Enzymol. 433: 113-2610.1016/S0076-6879(07)33006-1.

Paik, I.Y., Jeong, M.H., Jin, H.E., Kim, Y.I., Suh, A.R., Cho, S.Y., et al. 2009. Fluid replacement following dehydration reduces oxidative stress during recovery. Biochem. Biophys. Res.

Commun. 383: 103-710.1016/j.bbrc.2009.03.135.

Penkman, M.A., Field, C.J., Sellar, C.M., Harber, V.J., and Bell, G.J. 2008. Effect of hydration status on high-intensity rowing performance and immune function. Int. J. Sports Physiol. Perform. 3: 531-46

Powers, S.K., Ji, L.L., and Leeuwenburgh, C. 1999. Exercise training-induced alterations in skeletal muscle antioxidant capacity: a brief review. Med. Sci. Sports Exerc. 31: 987-97 
Powers, S.K., Radak, Z., and Ji, L.L. 2016. Exercise-induced oxidative stress: past, present and future. J. Physiol.10.1113/JP270646.

Sallam, N. and Laher, I. 2016. Exercise Modulates Oxidative Stress and Inflammation in Aging and Cardiovascular Diseases. Oxid. Med. Cell. Longev. 2016: 723963910.1155/2016/7239639. Sayre, L.M., Lin, D., Yuan, Q., Zhu, X., and Tang, X. 2006. Protein adducts generated from products of lipid oxidation: focus on HNE and one. Drug Metab. Rev. 38: 651$7510.1080 / 03602530600959508$.

Schliess, F. and Haussinger, D. 2002. The cellular hydration state: a critical determinant for cell death and survival. Biol. Chem. 383: 577-8310.1515/BC.2002.059.

Sommerfield, L.M., McAnulty, S.R., McBride, J.M., Zwetsloot, J.J., Austin, M.D., Mehlhorn, J.D., et al. 2015. Validity of Urine Specific Gravity when Compared to Plasma Osmolality as a Measure of Hydration Status in Male and Female NCAA Collegiate Athletes. J. Strength Cond. Res.10.1519/JSC.0000000000001313.

Sureda, A., Mestre-Alfaro, A., Banquells, M., Riera, J., Drobnic, F., Camps, J., et al. 2015. Exercise in a hot environment influences plasma anti-inflammatory and antioxidant status in well-trained athletes. J. Therm. Biol. 47: 91-810.1016/j.jtherbio.2014.11.011. Villano, D., Fernandez-Pachon, M.S., Troncoso, A.M., and Garcia-Parrilla, M.C. 2004. The antioxidant activity of wines determined by the ABTS $(+)$ method: influence of sample dilution and time. Talanta 64: 501-910.1016/j.talanta.2004.03.021.

Wadley, A.J., Turner, J.E., and Aldred, S. 2016. Factors influencing post-exercise plasma protein carbonyl concentration. Free Radic. Res. 50: 375-8410.3109/10715762.2015.1131824. 


\section{Table 1. Subject Characteristics}

\begin{tabular}{lll}
\hline Descriptive & Males $(\mathbf{n}=\mathbf{5 6})$ & Females $(\mathbf{n}=\mathbf{2 6})$ \\
\hline Height $(\mathrm{m})$ & $1.75( \pm 0.01)^{*}$ & $1.64( \pm 0.01)$ \\
Age $(\mathrm{yrs})$ & $19.3( \pm 0.2)$ & $19.8( \pm 0.3)$ \\
Experience $(\mathrm{yrs})$ & $9.6( \pm 0.5)$ & $15.1( \pm 0.3)^{*}$ \\
Weight $(\mathrm{kg})$ & $78.1( \pm 1.8)^{*}$ & $62.2( \pm 1.2)$ \\
Percent fat & $13.9( \pm 0.6)$ & $20.3( \pm 0.7)^{*}$ \\
\hline
\end{tabular}

*Denotes statistical significance at $\mathrm{p} \leq 0.001$. All values are mean $\_$SD. 
Table 2.

Significant

Correlations

\begin{tabular}{|c|c|c|c|}
\hline 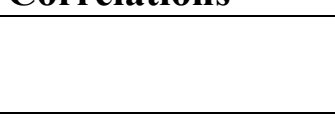 & $\begin{array}{c}\text { Pre- } \\
\text { Dehydration } \\
\end{array}$ & $\begin{array}{c}\text { Immediate Post } \\
\text { dehydration }\end{array}$ & $\begin{array}{l}\text { 1-hr Post Full } \\
\text { Rehydration }\end{array}$ \\
\hline $\begin{array}{l}\text { F2-isoprostanes } \\
\text { and FRAP }(n=24)\end{array}$ & & & $\mathrm{p} \leq 0.005,-0.587$ \\
\hline $\begin{array}{l}\text { Plasma } \\
\text { Osmolality and } \\
\text { TEAC }(n=33)\end{array}$ & & $\mathrm{p} \leq 0.02,-0.404$ & $\mathrm{P} \leq 0.037,-0.376$ \\
\hline
\end{tabular}




\section{Figure Legends}

Figure 1. Concentrations of plasma osmolality $(n=80)$ pre-exercise and throughout the dehydration and rehydration protocol. ${ }^{* *}$ pre-exercise versus immediate post-exercise $(\mathrm{p} \leq 0.01)$, * immediate post-exercise versus 1 -hr post full rehydration. All values are expressed as mean \pm SD.

Figure 2. Trolox Equivalent Antioxidant Capacity (TEAC) $(n=33)$ pre-exercise and throughout the dehydration and rehydration protocol. $* * *$ pre-exercise versus immediate post-exercise $(\mathrm{p} \leq 0.001), \quad * *$ pre-exercise versus 1 -hr post full rehydration $(\mathrm{p} \leq 0.001)$ and immediate postexercise versus 1 -hr post full rehydration $(\mathrm{p} \leq 0.001)$. All values are expressed as mean \pm SD.

Figure 3. Ferric Reducing Ability of Plasma (FRAP) (n=76) pre-exercise and throughout the dehydration and rehydration protocol. $* * *$ pre-exercise versus immediate post-exercise $(\mathrm{p} \leq 0.001), \quad * *$ pre-exercise versus $1-\mathrm{hr}$ post full rehydration $(\mathrm{p} \leq 0.05)$ and immediate postexercise versus 1 -hr post full rehydration $(\mathrm{p} \leq 0.001)$. All values are expressed as mean \pm SD.

Figure 4. Concentration of protein carbonyls $(n=24)$ pre-exercise and throughout the dehydration and rehydration protocol. $* *$ pre-exercise versus $1-h r$ post full rehydration $(\mathrm{p} \leq 0.01)$. All values are expressed as mean $\pm \mathrm{SD}$.

Figure 5. Concentration of $\mathrm{F}_{2}$-isoprostanes $(\mathrm{n}=24)$ pre-exercise and throughout the dehydration and rehydration protocol. $* * *$ pre-exercise versus immediate post-exercise $(\mathrm{p} \leq 0.001), \quad * *$ preexercise versus 1 -hr post full rehydration $(\mathrm{p} \leq 0.001)$ and immediate post-exercise versus 1 -hr post full rehydration $(\mathrm{p} \leq 0.001)$. All values are expressed as mean $\pm \mathrm{SD}$. 


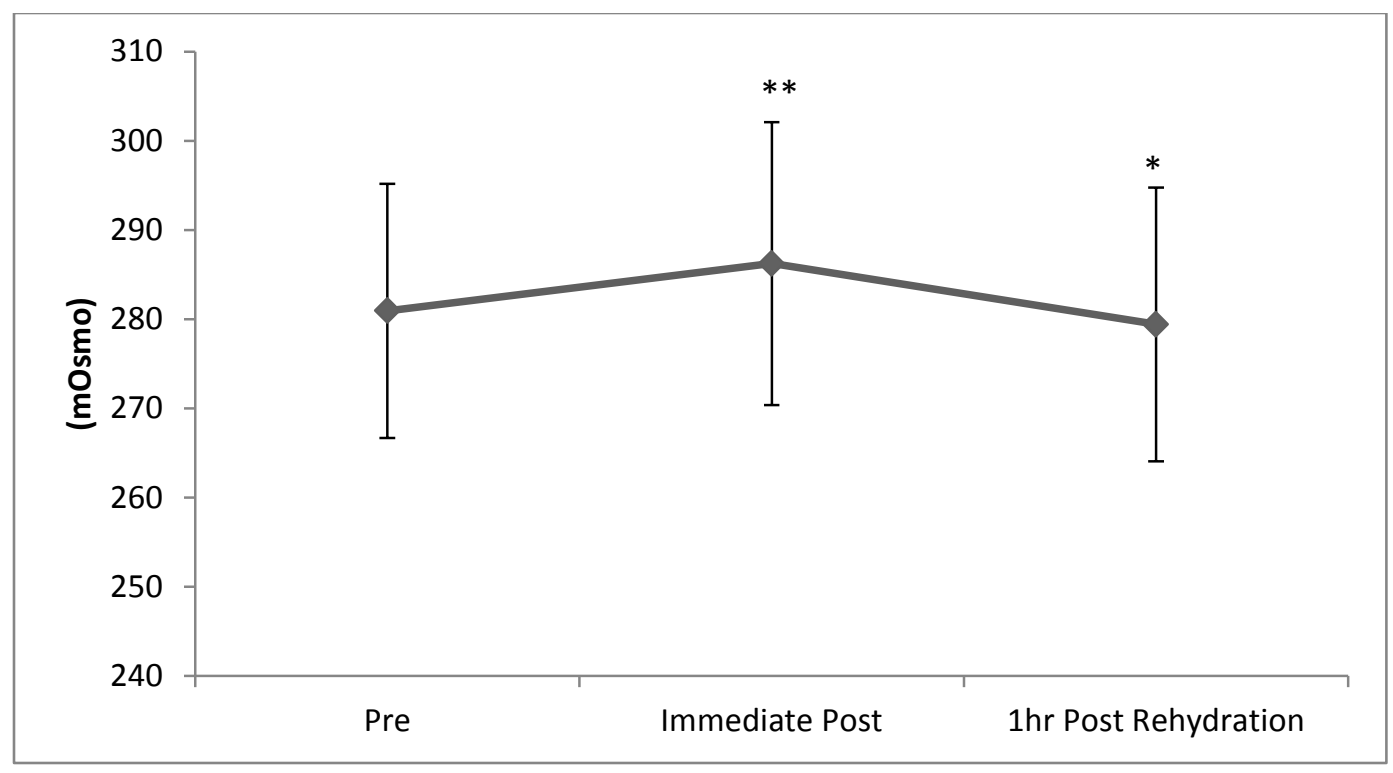


22

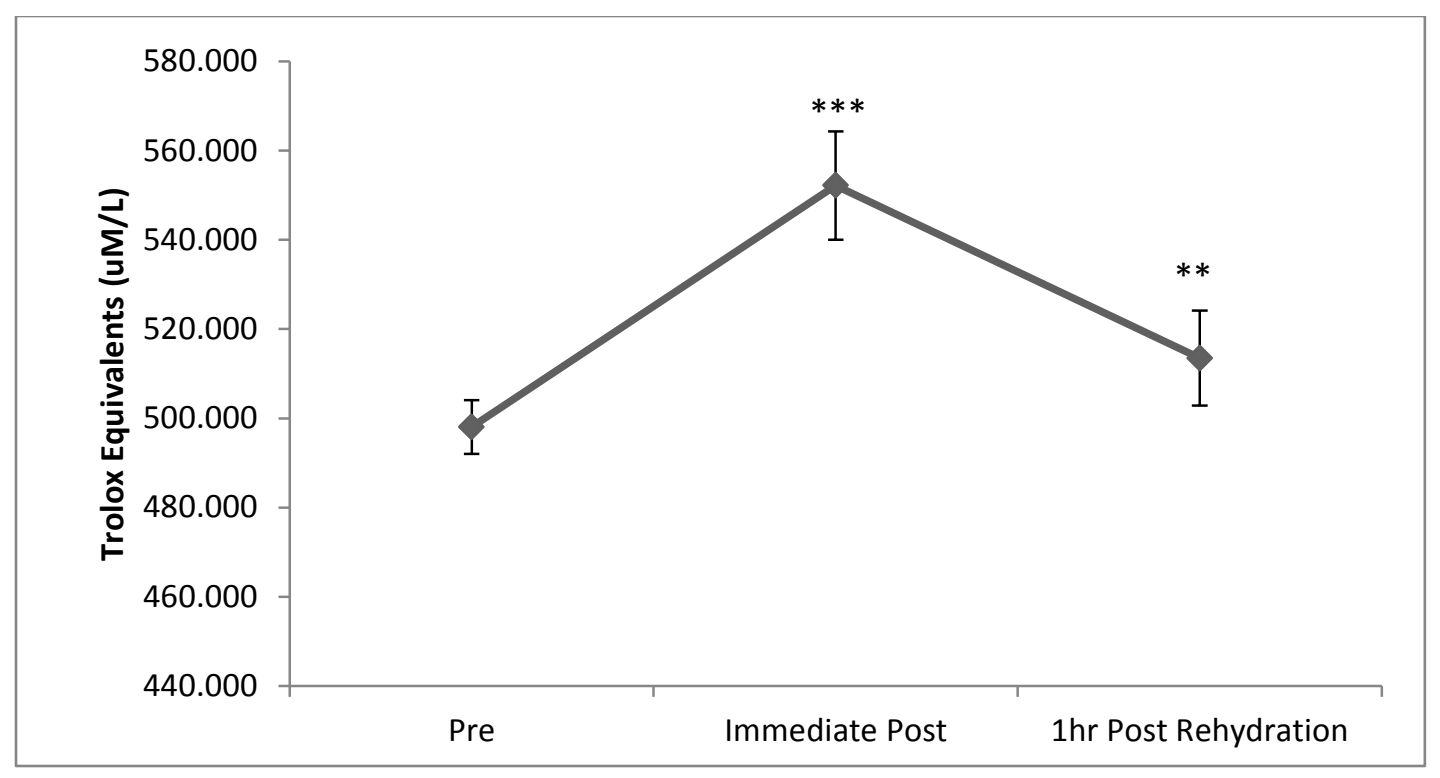


23

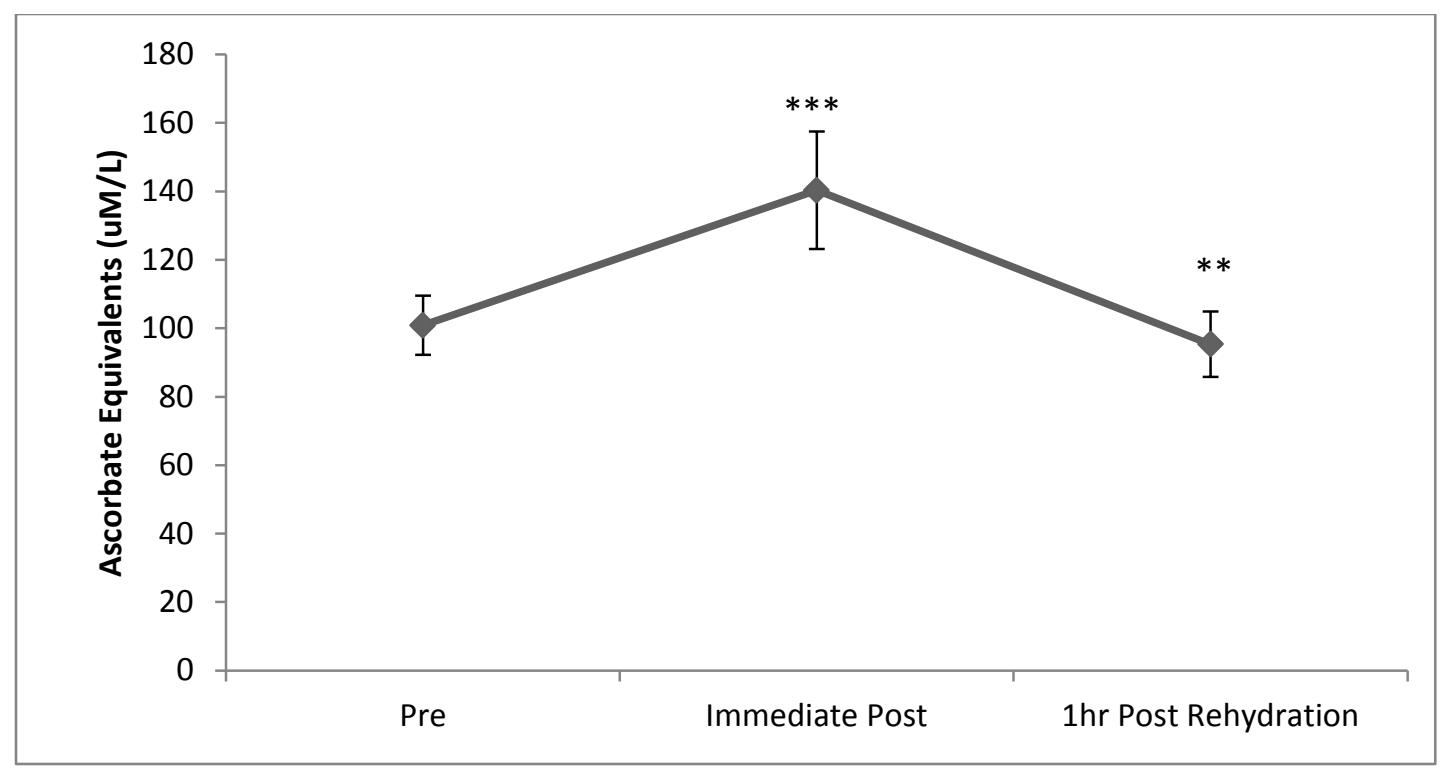

https://mc06.manuscriptcentral.com/apnm-pubs 
24

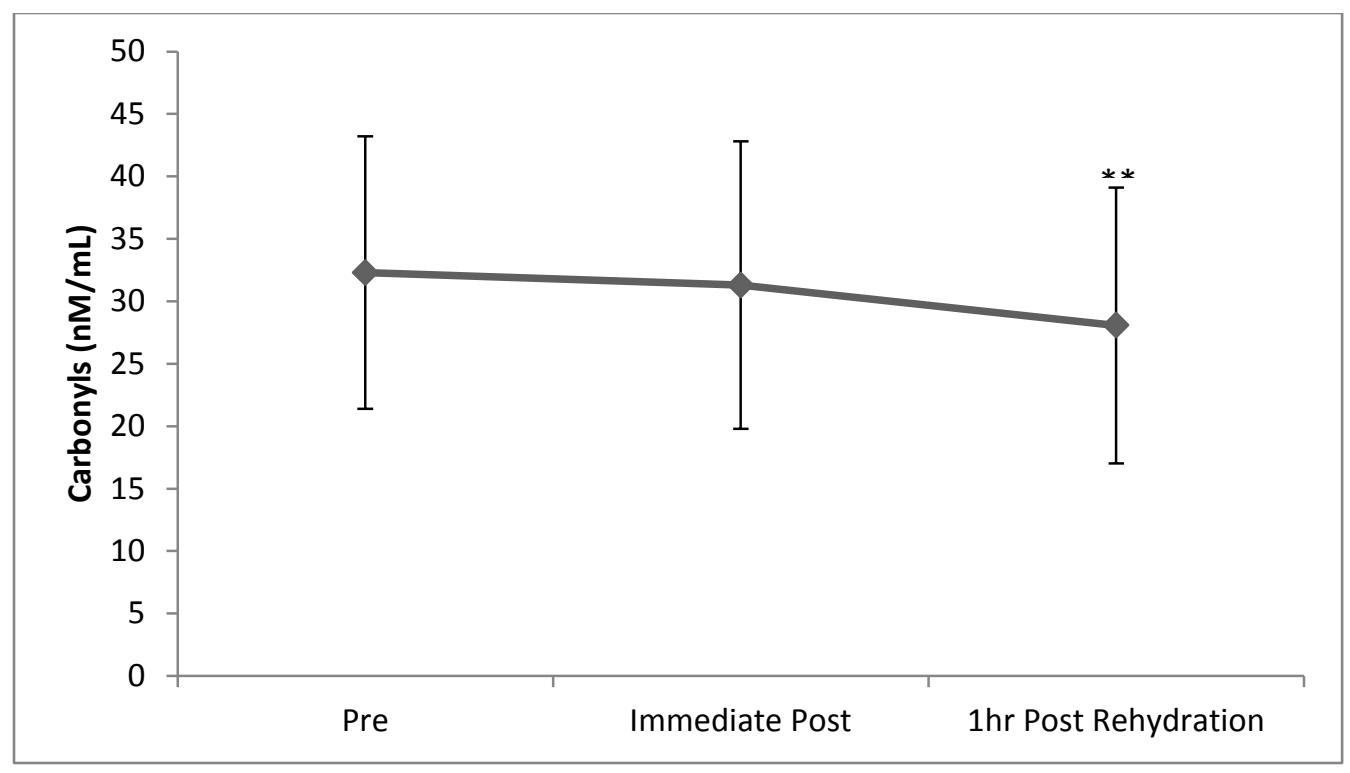

https://mc06.manuscriptcentral.com/apnm-pubs 
25

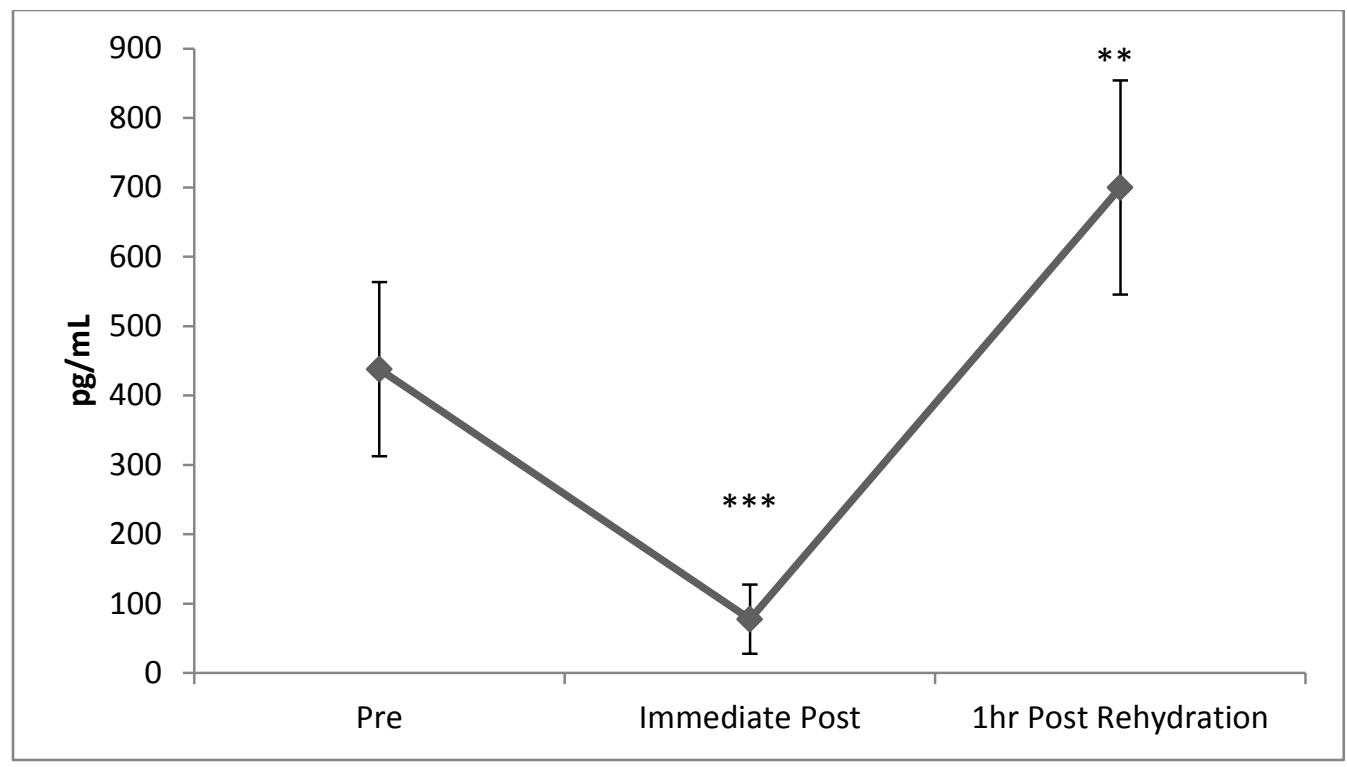

https://mc06.manuscriptcentral.com/apnm-pubs 